\title{
Depression and Its Risk Factors Among Patients with Chronic Obstructive Pulmonary Disease (COPD) in a Tertiary Level Hospital
}

\author{
U K Barua ${ }^{1}$, D K Ghosh ${ }^{2}$, A Biswas ${ }^{3}, \mathrm{M} \mathrm{Nath}^{4}, \mathrm{~B} \mathrm{Biswash}^{5}$, N saha ${ }^{6}, \mathrm{M} \mathrm{Karim}^{7}$
}

\begin{abstract}
Background: COPDis accompanied with several co-morbidities among which depression is a major one. The aim of the study is to assess the prevalence of depression and associated risk factors in patients with COPD in a tertiary level hospital of Dhaka city, Bangladesh. Method: This Cross-sectional study was carried out the Department of Respiratory Medicine of ShaheedShurawardy Medical College Hospital from January 2014 to June 2014. 317 COPD patients were selected by systematic sampling. A questionnaire was administered among the respondents to collect the data regarding their socio-demographic conditions followed by Patient Health Questionnaire 9 (PHQ-9) to measure the level of depression. Result: Among the 317 respondents, the mean age was 58.40 and $98.1 \%$ were male, $1.9 \%$ were female. The proportion of depression among patients with COPD was $81.6 \%$ (8 out of 10 COPD patients). Among the all respondents, $23.3 \%$ had moderate depression, $14.5 \%$ had moderately severe depression, and only $4.7 \%$ had severe depression. The risk factors which were found to be involved with the development of depression among COPD patients were stage 2 and stage 3 COPD, onset of COPD $\geq 40$ years of age, literate respondents. Conclusion: The study highlight the importance of routine screening for depression of all COPD patients in all healthcare settings and implementation of strategies for proper management and prevention of depression in those patients. [J Shaheed Suhrawardy Med Coll 2015;5(2): 44-48]
\end{abstract}

Keywords: COPD, Depression, PHQ-9

\section{Introduction}

Chronic obstructive pulmonary disease (COPD) is a leading cause of morbidity and mortality in countries of high, middle, and low income. Estimates from WHO's Global Burden of Disease and Risk Factors projectl show that in 2001, COPD was the fifth leading cause of death in high-income countries, accounting for $3.8 \%$ of total deaths, and it was the sixth leading cause of death in nations of low and middle income, accounting for $4.9 \%$ of total deaths. In this same report, COPD was also estimated to be the seventh and tenth leading cause of disability-adjusted life years in countries of high income and in those of low or middle income, respectively ${ }^{1}$. The burden of COPD in Asia is currently greater than that in developed Western countries linked to the epidemic of tobacco exposure and indoor and outdoor air pollution in
Asian countries. Total deaths from COPD are projected to increase by more than $30 \%$ in the next 10 years without interventions to cut risks, particularly exposure to tobacco smoke $^{2}$.

In Bangladesh, prevalence rates of COPD vary from 2 to 22 $\% 3$. There were some variations in prevalence rates depending upon the place of residence and socio-economic groups but significant differences were observed in relation to the smoking habit and exposures to combustion of solid fuels and environmental tobacco smoke. More than $80 \%$ of these patients are not diagnosed and therefore they are not treated appropriately.

The natural course of COPD is complicated by the development of systemic consequences and co-morbidities ${ }^{4}$. These may be major features in the clinical presentation of

1. Dr. Uttam Kumar Barua, Professor \& Head, Department of Respiratory Medicine, ShaheedSuhrawardy Medical College \& Hospital, Dhaka.

2. Dr. Dilip Kumar Ghosh, Assistant Professor, Department of Gastroenterology, ShaheedSuhrawardy Medical College \& Hospital, Dhaka.

3. Dr. Amar Biswas, Assistanmt Professor, Department of Respiratory Medicine, ShaheedSuhrawardy Medical College \& Hospital, Dhaka.

4. Dr. MuktaNath, Emergency Medical Officer, ShaheedSuhrawardy Medical College \& Hospital, Dhaka.

5. Dr. BipulKantiBiswas, Assistanmt Professor, Department of Respiratory Medicine, NIDCH, Dhaka.

6. Dr.NiharRanjanSaha, Assistanmt Professor, Department of Respiratory Medicine, NIDCH, Dhaka.

7. Dr. MesbahulKarim Ruble, Assistanmt Professor, Department of Respiratory Medicine, ShaheedSuhrawardy Medical College \& Hospital, Dhaka.

Correspondence

Dr. Uttam Kumar Barua, Professor \& Head, Department of Respiratory Medicine, ShaheedSuhrawardy Medical College \& Hospital, Dhaka.

Cell: 01819402358.

Conflict of interest: No conflict of interest

Contributions by authors: All authors contributed form protocol to write up of the article 
COPD. Systemic consequences may be defined as non-pulmonary manifestations of COPD with an immediate cause-and-effect relationship, whereas co-morbidities are diseases associated with COPD. Depression is regarded as one of the major co-morbidities of COPD ${ }^{4}$. Evidence from a study has shown that depression is related to the outcome of emergency treatment in patients with COPD $^{5}$. A few studies were conducted to find out the prevalence of depression among COPD patients and the results were variable.

In India, a number of studies were conducted to find out the prevalence of depression in population and a study from urban South India estimated the prevalence of depression is $25.7 \%$ among the people above 60 years of age ${ }^{6}$.

A study conducted in Bhopal demonstrated the prevalence of depression among patients with COPD patients is $72 \%{ }^{7}$.A systemic review conducted on different studies remains inconclusive regarding the prevalence of depression in patients with $\mathrm{COPD}^{8}$. The proportion of depression among the patients with COPD can demonstrate how depression can affect the life of a patient with chronic obstructive pulmonary disease ${ }^{4}$.

A study in India explained that the prevalence of depression among the patients with COPD is extremely increased and it points that over eight in each ten respondents were tormented by depression. The prevalence of depression of this study was in line with results of the study conducted by Dey12 in Madhyapradesh. In this study it was gotten that nearly seven out of ten COPD patients were depressed ${ }^{9}$.

Tze-Pinnanogram focused in his study that depression is related to inflated mortality risk for the COPD patients including longer hospitalization, persistent smoking behavior, inflated symptom burden, poorer physical and social functioning, and reduced quality of life.Trials of antidepressant drug and psychological interventions ought to offer conclusive proof of improved survival, quality of life, and self-management behavior and reduced health care utilization $^{10}$.

To improve this depression for COPD patient's rehabilitation programmers can embrace up to a few sessions per week of progressive and supervised exercise, along with education and psychosocial support, considerably cut back anxiety and depression over normal care in patients with COPD $^{11}$.

In Bangladesh no study available regarding depression with COPD patients so there is still a gap of knowledge regarding the proportion of depression among patients with COPD. Therefore, the present study was designed to find out the Depression and its risk factors among patients with chronic obstructive pulmonary disease (COPD) in a tertiary level hospital of Dhaka city, Bangladesh.

\section{Methodology}

This Cross-sectional study was carried out the Department of Respiratory Medicine of ShaheedSuhrawardy Medical College Hospital in Dhaka city from January 2014 to June 2014. Diagnosed COPD patient of either sex aged 18years and above were included in this study. Respondents not willing to participate in the study were excluded from the study. The sample of the study was 317 respondents who fulfilled the inclusion and exclusion criteria. Systematic sampling technique was adopted for this study. The data was collected by face to face interview of the respondents who were suffering from COPD and met the selection criteria. Data collection tool was Patient Health Questionnaire (PHQ-9) and Patient complete PHQ-9 Quick Depression Assessment. All the relevant collected data was compiled on a master chart first and then statistical analysis of the results was obtained by using window based computer software devised with Statistical Packages for Social Sciences (SPSS-17) (SPSS Inc, Chicago, IL, USA). The result was presented in tables, figures, diagrams. Qualitative data were expressed as frequency and percentage and quantitative data were expressed as mean and standard deviation. The significance of individual symptoms and combined symptoms were analyzed by applying a multivariate logistic regression model adjusting for age and gender. The result was presented as odd ratios with $95 \%$ confidence intervals. A two- sided $\mathrm{p}$ value less than 0.05 was regarded as statically significant, and $95 \%$ confidence intervals was computed using a logistic regression model. Prior to the commencement of this study, the research protocol was approved by the Ethical review committee of BMRC, Dhaka.

\section{Results:}

The study population consisted of 317 persons, 98.1\% male and $1.9 \%$ female. The majority $(33.4 \%)$ of the study population belonged to old age group and the mean age of them was calculated to be 58.4 years (Table-1\&2). Nearly $98.7 \%$ of the sample population was married. In regards to the occupation of the respondents majority was from Small Business man (22.4\%), about $12.6 \%$ from Agricultural workers and about $65 \%$ constituted other occupation like, Govt. and Non-Govt. employee, Industrial worker, Rice mill worker, Driver, Day laborer, Housewife, self-employed, etc. (Table-3). With respect to the educational background, about $22.4 \%$ of the respondents were informal educated and the next highest group $17 \%$, primary school not completed. The highest literate group (13.2\%) belonged to Graduate and above. About $59 \%$ of the respondents reported to earn a monthly income of Tk. 10,000-19,999 with an average income Tk. 7,500 (Table- 4). Half of the respondents (49.5\%) were found to be Ex-smoker and nearly half of the respondents were Regular smoker(47\%) and only $3.5 \%$ of the respondents were Non-smoker(Table-5).

Table 1: Distribution of respondents by age

\begin{tabular}{lcc}
\hline Age & Frequency & Percent \\
\hline $35-44$ & 33 & 10.4 \\
$45-54$ & 72 & 22.7 \\
$55-64$ & 106 & 33.4 \\
$>=65$ & 106 & 33.4 \\
Total & $\mathbf{3 1 7}$ & $\mathbf{1 0 0 . 0}$ \\
\hline
\end{tabular}

Mean $( \pm \mathrm{SD})$ age is $58.4( \pm 11.8)$ years within range 35 - 95 years 
Table 2: Distribution of respondents by gender

\begin{tabular}{lcc}
\hline Gender & Frequency & Percent \\
\hline Male & 311 & 98.1 \\
Female & 6 & 1.9 \\
Total & $\mathbf{3 1 7}$ & $\mathbf{1 0 0 . 0}$
\end{tabular}

Table 3: Distribution of respondents by educational level

\begin{tabular}{lcc}
\hline Educational level & Frequency & Percent \\
\hline Informal education & 71 & 22.4 \\
Primary school not completed & 54 & 17.0 \\
Primary & 35 & 11.0 \\
Secondary school not completed & 34 & 10.7 \\
Secondary & 26 & 8.2 \\
Higher secondary not completed & 19 & 6.0 \\
Higher secondary & 36 & 11.4 \\
Graduate and above & 42 & 13.2 \\
Total & $\mathbf{3 1 7}$ & $\mathbf{1 0 0 . 0}$ \\
\hline
\end{tabular}

Table 4: Distribution of respondents by occupation status

\begin{tabular}{lcc}
\hline Occupational status & Frequency & Percent \\
\hline Student & 13 & 4.1 \\
Government employee & 30 & 9.5 \\
Non-government employee & 4 & 1.3 \\
Business (small) & 71 & 22.4 \\
Business (large) & 6 & 1.9 \\
Farming (landowner and farmer) & 18 & 5.7 \\
Agriculture worker & 40 & 12.6 \\
Industrial worker & 24 & 7.6 \\
Rice mill worker & 1 & .3 \\
Rickshaw puller & 14 & 4.4 \\
Driver & 8 & 2.5 \\
Daily laborer & 13 & 4.1 \\
Homemaker/housewife & 4 & 1.3 \\
Self employed & 6 & 1.9 \\
Retired & 23 & 7.3 \\
Unemployed, able to work & 5 & 1.6 \\
Unemployed, not able to work & 28 & 8.8 \\
Others & 9 & 2.8 \\
Total & $\mathbf{3 1 7}$ & $\mathbf{1 0 0 . 0}$ \\
\hline
\end{tabular}

Table 5: Distribution of respondents by smoking status

\begin{tabular}{lcc}
\hline Smoking status & Frequency & Percent \\
\hline Regular smoker & 149 & 47.0 \\
Non-smoker & 11 & 3.5 \\
Ex-smoker & 157 & 49.5 \\
Duration of smoking & & \\
$\quad$ Less than 10 years & 47 & 15.4 \\
$\quad$ More than 10 years & 259 & 84.6 \\
Total & $\mathbf{3 1 7}$ & $\mathbf{1 0 0 . 0}$
\end{tabular}

The proportion of depression among patients with COPD was $81.6 \%$. Among the all respondents, $23.3 \%$ had moderate depression, $14.5 \%$ had moderately severe depression, and only $4.7 \%$ had severe depression. It was also found that the depression increases with the progression of the stages of COPD. The risk factors which were found to be involved with the development of depression among COPD patients were stage $2 \mathrm{COPD}(\mathrm{OR}=0.094, \mathrm{p}=0.001)$ and stage 3 $\mathrm{COPD}(\mathrm{OR}=0.206, \mathrm{p}=0.001)$, onset of $\mathrm{COPD} \geq 40$ years of age $(\mathrm{OR}=0.368, \mathrm{p}=0.001)$, literate $(\mathrm{OR}=13.722, \mathrm{p}=0.001)$ (Table-15\&16).

Table 6: Level of depression among the respondents

\begin{tabular}{lcc}
\hline Patients health status & Frequency & Percent \\
\hline Nil & 71 & 22.4 \\
Minimal depression & 39 & 12.3 \\
Mild depression & 72 & 22.7 \\
Moderate depression & 74 & 23.3 \\
Moderately severe depression & 46 & 14.5 \\
Severe depression & 15 & 4.7 \\
Total & $\mathbf{3 1 7}$ & $\mathbf{1 0 0 . 0}$ \\
\hline
\end{tabular}

Table 7: Strength of association of a set of independent variables with status of depression

\begin{tabular}{lcccc}
\hline Independent variables & \multirow{2}{*}{ OR } & p- value & \multicolumn{2}{c}{ 95\% CI for Exp (B) } \\
\cline { 4 - 5 } & & & Lower & Upper \\
\hline & & & & \\
Age category & 1.000 & & & \\
Age group (35-44) & 0.722 & 0.456 & 0.307 & 1.700 \\
Age group (45-54) & 0.196 & 0.001 & 0.078 & 0.493 \\
Age group (55-64) & 0.426 & 0.046 & 0.185 & 0.985 \\
Age group ( $\geq 65)$ & & & & \\
Educational status & 1.000 & & & \\
Illiterate & & & & \\
Literate & 13.722 & 0.001 & 3.274 & 57.503 \\
Alcohol consumption status & & & & \\
Alcohol consumer & 1.000 & & & \\
Teetotaler & 2.240 & 0.292 & 0.500 & 10.037 \\
& & & & \\
\hline
\end{tabular}




\begin{tabular}{lcccc}
\hline \multirow{2}{*}{ Independent variables } & \multirow{2}{*}{ OR } & p- value & \multicolumn{2}{c}{ 95\% CI for Exp (B) } \\
\cline { 4 - 5 } & & & Lower & Upper \\
\hline Hypertension & & & & \\
Absent & 1.000 & & \\
Present & 0.223 & 0.005 & 0.078 & 0.639 \\
Stage of COPD & & & & \\
Stage 1 & 1.000 & & \\
Stage 2 & 0.094 & 0.001 & 0.042 & 0.207 \\
Stage 3 & 0.206 & 0.001 & 0.093 & 0.457 \\
Stage 4 & 0.711 & 0.390 & 0.327 & 1.547 \\
Age of onset of COPD & & & & \\
$<40$ years & 1.000 & & \\
$\geq 40$ years & 0.368 & 0.002 & 0.194 & 0.697 \\
Family history of COPD & & & & \\
Absent & 1.000 & & & \\
Present & 0.960 & 0.937 & 0.343 & 2.682 \\
Constant & 0.00 & 0.000 & & \\
\hline
\end{tabular}

\section{Discussion}

Among the major non communicable diseases, COPD is the leading cause of morbidity and mortality and prevalence of COPD is rising particularly in the developing world. Co-morbid psychiatric illness accompanied with COPD can be a unique challenge in the treatment of COPD. The presence of unrecognized depression in patients with COPD can be a major concern, as they are at risk of developing major depression and may increase the burden of physical activity which turns into less productivity and poor quality of life.

The current study shows that the prevalence of depression among the COPD patients is very high and it indicatrs that more than 8 in every 10 respodents were suffering from depression. The prevalence of depression of the current study was in line with result of the study conducted by Dey $^{12}$ in Madhya Pradesh, which found that almost 7 out of 10 COPD patients were depressed. The same screening tool was used in the study and the mean age (58.7 9.6 years) of the respondent of that study was nearly similar to the mean age 58.4 year of the respondents of the current study. Kuniket al. ${ }^{12}$ in the USA also found that 8 out of patients with chronic breathing disorders had depression, anxiety or both. However that study included all chronic breathing disorders, namely, COPD, asthma and bromchiectasis. Solaniet at. ${ }^{13}$ found the prevalence of depression to be $71.0 \%$ and that is comparable with result current study.

The proportion of depression among the COPD patients, found in our study varied from the findings of Manenet al. ${ }^{14}(25 \%)$ and wagena et al. ${ }^{16}(22 \%)$-this may be due to the socio-cultural and economic differences between the Dutch population and Indian population and different healthcare delivery system. It is also to be mentioned that Manenet at. ${ }^{14}$ used centers for epidemiologic studies Depression (CES-D) scale 22 and Wagena et at. ${ }^{16}$ used dutch version of SCL-90-R23 for measuring the depression level.
The present study found that depression increased significantly with the progression of COPD. Apart from it, the risk indicators which are determined from the current study are educational level, income status, age of onset of COPD. There are not much studies focusing on the risk indicators of depression among COPD patients in India and the only study Dey ${ }^{12}$ found that depression increases significantly with the progression of COPD and the finding corresponds with the present study. Manenet. al. ${ }^{14}$ found that patients with mild to moderate COPD severity are not at increased risk for depression but patient with severe COPD had higher risk of depression. However, Wagena et al. did not find any significant association between severity of COPD and of depression.

The risk factors found in the study of Schaneet al. were female gender, marital status, educational status, co-morbid diabetes, arthritis and difficulty in walking. This study was done in among the US population who were 50 year of age. The current study had only 6 female respondents and hence no valid comparison could be done regarding the gender. Among the other risk factors, educational level was also found to be a risk indicator of our study. The variation among the risk factors of these two studies may be due to the difference of culture, economic condition and health-care facilities of the US population and Bangladeshi population. Manenet al. ${ }^{14}$ also found that living also was a risk factor of depression among patients with COPD in dutch population. This study, did not find significant association of age, sex, education and co-morbidty with depression among COPD. These findings are in line with the findings for the current study.

The study has a number of limitations. Being a hospital-based study, finding of this study may not be generalized and it may not give the actual proportion of depression among patients with COPD of the whole country.

Moreover, diagnosis of COPD and staging of COPD were done by spiro metric test and the results were varied form different examiner's motivation and instructions provided to the patients. This may be resulted inter-examiner variation, which was not calculated. Recognition of co-morbid depression is difficult, because some of the physical symptoms of COPD may mimic the core symptoms of depression, for example, poor sleeping pattern, anorexia and loss of enjoyment due to breathlessness which may lead to overestimation of depression among COPD patients.

\section{Conclusion}

The study found that the proportion of depression among patients with COPD is $81.6 \%$ (8 out of 10 patients) and it can be considered very high proportion. The study also confirmed the risk factors of developing depression in COPD patients were onset of COPD $\geq 40$ years of age, literate respondents, stage 2 and stage 3 COPD. This Study highlight the importance of routine screening for depression of all COPD patients in all healthcare settings and implementation of strategies for proper management and prevention of depression in those patients. Large scale studies should be conducted to examine the prevalence of 
depression among COPD patients and their risk factors in the other parts of the country. Awareness regarding depression among COPD patients should be enhanced especially for the health care professionals. Above all, national management guidelines for the depression among COPD patients should be developed and implemented.

\section{References}

1. Lopez AD, Mathers CD, Ezzati M, Jamison DT, Murray CJL. Global burden of disease and risk factors. Washington: The World Bank, 2006. 2. WHO. The global burden of disease: 2004 up-date. Geneva: World Health Organization, 2008.

3. National guideline

4. Decramer M, Rennard S, Troosters T, Mapel DW, Giardino N, Mannino D, Wouters E Sethi S, Cooper CB. COPD as a lung disease with systemic consequences-clinical impact, mechanism, and potential for early intervention. COPD 2008;5:235-56.

5. DahlénInger, JansonChrister Anxiety and Depression Are Related to the Outcome of Emergency Treatment in Patients With Obstructive Pulmonary Disease. Chest 2002;122:1633-7.

6. Poongothai S, Pradeepa R, Ganesan A, Mohan V. Prevalence of depression in a large urban south Indian population: the Chennai Urban Rural Epidemiology Study (Cures-70). PLoS ONE 4(9): e7185.

7. Dey S. Prevalence of depression in stable chronic obstructive pulmonary disease. Indian J Chest Dis Allied Sci2011 Jan-Mar;53:35-9.
8. L van Ede, C J Yzermans, H J Brouwer, Preva-lence of depression in patients with chronic ob-structive pulmonary disease: a systematic review, Thorax 1999;54:688-92.

9. Kalyan Bhowmik, M. A. (2012). Prevalence of depression and its risk factors among patients with chronic obstructive pulmonary disease in a tertiary level hospital in West Bengal, India. South East Asia Journal Of Public Health, 34-40.

10. Tze-Pin Ng, M. N.-C. (2007). Depressive Symptoms and Chronic Obstructive Pulmonary Disease. Archives of Internal Medical, 60-67. 11. Peter A. Coventrya, D. H. (2007). Comprehensive pulmonary rehabilitation for anxiety and depression in adults with chronic obstructive pulmonary disease:Systematic review and meta-analysis. Journal of Psychosomatic Research, 551-565.

12. Dey S. Prevalence of depression in stable chronic obstructive pulmonary disease. Indian J Chest Dis Allied Sci 2011 Jan-Mar;53:35-9. 13. Kunik ME, Roundy K, Veazey C, Souchek J, Richardson P, Wray NP, et al. Surprisingly high prevalence of anxiety and depression in chronic breathing disorders. Chest 2005;127:1205-11.

14. Solano JP, Gomes B, Higginson IJ.A comparison of symptom prevalence in far advanced cancer, AIDS, heart disease, and chronic obstructive pulmonary disease and renal disease. J Pain SymptomManage 2006;31:58-69.

15. Manen JGV, Bindels PJE, Dekker FW, Jzermans CJI, Zee JSV, Schade E. Risk of depression in patients with chronic obstructive pulmonary disease and its determinants. Thorax 2002; 57:412-6. 